\title{
First report of Cytauxzoon sp. infection in a domestic cat from Portugal
}

\author{
Ana Margarida Alho', Joana Silva², Maria João Fonseca ${ }^{3}$, Filipa Santos ${ }^{3}$, Cláudia Nunes ${ }^{2}$, Luís Madeira de Carvalho ${ }^{1}$, \\ Manuel Rodrigues ${ }^{2}$ and Luís Cardoso ${ }^{4^{*}}$
}

\begin{abstract}
Background: Cytauxzoonosis is an emerging and life-threatening tick-borne feline disease caused by haemoprotozoan parasites of the genus Cytauxzoon. Information regarding epidemiological and clinical presentation of infections by species other than Cytauxzoon felis is scant. A case of Cytauxzoon sp. infection is described in a 2-year-old mixed breed male domestic cat from Portugal, presenting a history of acute lethargy, anorexia and pyrexia.

Results: Complete blood count revealed a severe anaemia, leucocytosis and thrombocytopenia. A pleural effusion was noticed on thoracic radiograph, and marked splenomegaly and free abdominal fluid were visualized by ultrasound. A molecular screening for the detection of causative agents of infectious anaemia was performed, and a positive result for Piroplasmorida was obtained. DNA sequencing of a $743 \mathrm{bp}$ amplicon of the $18 \mathrm{~S}$ rRNA gene (GenBank accession no. KU710344) revealed $99.9 \%$ identity with Cytauxzoon manul.

Conclusions: This is the first report of Cytauxzoon sp. (clustering together with C. manul) in a felid from Portugal. Clinical manifestations along with molecular analysis suggest the hypothesis that domestic cats might be infected with and serve as a reservoir host for C. manul.
\end{abstract}

Keywords: Cytauxzoon sp., Cytauxzoon manul, Haemoparasite, Piroplasm, Domestic cat, Tick-borne disease, Portugal

\section{Background}

Feline vector-borne diseases are being increasingly reported worldwide. Several factors have been linked to this sharp expansion and wide distribution range, namely climate changes, enhanced international commerce and global transport, increased drug resistance among vectors and pathogens, demographic and political changes, and wildlife host abundance $[1,2]$.

Cytauxzoonosis is an emerging tick-borne feline disease caused by haemoprotozoan parasites of the genus Cytauxzoon (Theileriidae), with a few identified species [3]. This life-threatening pathological condition is characterized by a rapid course of illness and eventually death, usually in a couple of days. Cytauxzoon felis is the main agent of cytauxzoonosis, with different strains or genotypes capable of producing infection in domestic

\footnotetext{
* Correspondence: Icardoso@utad.pt

${ }^{4}$ Department of Veterinary Sciences, School of Agrarian and Veterinary Sciences, University of Trás-os-Montes e Alto Douro (UTAD), Vila Real, Portugal

Full list of author information is available at the end of the article
}

cats, lions and tigers $[4,5]$. A closely related piroplasm was reported in Pallas's cats (Otocolobus manul) from Mongolia and later described as a new species, Cytauxzoon manul, based on a significant sequence divergence $[6,7]$. Furthermore, C. manul has also been reported in African lions [8]; and Cytauxzoon sp. clustering together with $C$. manul in domestic cats [9-11], an Iberian lynx $[12,13]$, Eurasian lynxes and wildcats [14]. Experimental infection of domestic cats with blood from Pallas's cats infected with C. manul showed that they are susceptible to erythrocytic phases of this agent and presumably to other phases of the parasite's life-cycle [15]. Nevertheless, information regarding epidemiological distribution, clinical presentation, genetics and pathogenicity of infection by $C$. manul is scant. Besides, very little is known about species of Cytauxzoon other than C. felis, especially in Europe. Here, we report the first clinical case and molecular characterization of naturally occurring Cytauxzoon sp. infection in a domestic cat (Felis catus) from Portugal. 


\section{Clinical case}

In February 2015, a 2-year-old mixed breed intact male domestic cat (body condition score of 5/9) was presented to a referral veterinary hospital in Lisbon, with a history of acute onset of lethargy, anorexia and pyrexia. The cat was born and raised in the northern central region of Portugal) and had never travelled abroad. The animal had regular outdoor access and shared the house and backyard with its siblings. No health issues had previously been diagnosed, and its past history was unremarkable.

At physical examination the cat was severely depressed and had tachycardia, dyspnoea and tachypnoea. Body temperature was $40.0{ }^{\circ} \mathrm{C}$. Mucous membranes were pale and capillary refill time was $>2 \mathrm{~s}$. Blood was collected for a complete blood count (CBC), a serum chemistry profile and rapid tests for the detection of feline leukaemia virus (FeLV) antigen and of antibodies to feline immunodeficiency virus (FIV). CBC revealed a severe anaemia, leucocytosis and thrombocytopenia and routine serum biochemistry showed azotaemia and hyperbilirubinemia (Table 1). FIV and FeLV tests were negative.

To assess the cause of the dyspnoea and tachypnoea, lateral and ventrodorsal radiographic projections of the thorax were performed at full inspiration, revealing pleural effusion. Further abdominal ultrasound showed marked splenomegaly, kidneys with loss of definition and marked amount of anechoic (low cellularity) free fluid in the abdomen. Pleural and abdominal liquids collected and sent for cytology were compatible with a

Table 1 Complete blood count (CBC) and routine serum biochemistry results

\begin{tabular}{lll}
\hline Parameter & Values (unit) & Reference values (unit) \\
\hline Platelet count & $85 \times 10^{3} / \mathrm{mm}^{3}$ & $150-500 \times 10^{3} / \mathrm{mm}^{3}$ \\
Red blood cell count & $3.14 \times 10^{6} / \mathrm{mm}^{3}$ & $5.0-11.0 \times 10^{6} / \mathrm{mm}^{3}$ \\
White blood cell count & $19.6 \times 10^{3} / \mathrm{mm}^{3}$ & $5.5-19.5 \times 10^{3} / \mathrm{mm}^{3}$ \\
Alanine transaminase (ALT) & $46 \mathrm{U} / \mathrm{l}$ & $20-100 \mathrm{U} / \mathrm{l}$ \\
Albumin & $2.6 \mathrm{~g} / \mathrm{dl}$ & $2.2-4.4 \mathrm{~g} / \mathrm{dl}$ \\
Alkaline phosphatase (ALP) & $27 \mathrm{U} / \mathrm{l}$ & $10-90 \mathrm{U} / \mathrm{l}$ \\
Bilirubin & $0.7 \mathrm{mg} / \mathrm{dl}$ & $0.1-0.6 \mathrm{mg} / \mathrm{dl}$ \\
Blood urea nitrogen (BUN) & $66 \mathrm{mg} / \mathrm{dl}$ & $10-30 \mathrm{mg} / \mathrm{dl}$ \\
Chloride & $123 \mathrm{mmol} / \mathrm{l}$ & $107-120 \mathrm{mmol} / \mathrm{l}$ \\
Creatinine & $1.5 \mathrm{mg} / \mathrm{dl}$ & $0.3-2.1 \mathrm{mg} / \mathrm{dl}$ \\
Globulin & $3.7 \mathrm{~g} / \mathrm{dl}$ & $1.5-5.7 \mathrm{~g} / \mathrm{dl}$ \\
Glucose & $150 \mathrm{mg} / \mathrm{dl}$ & $70-150 \mathrm{mg} / \mathrm{dl}$ \\
Haematocrit & $16.0 \%$ & $24-45 \%$ \\
Haemoglobin & $4.9 \mathrm{~g} / \mathrm{dl}$ & $8.0-15.0 \mathrm{~g} / \mathrm{dl}$ \\
Potassium & $3.9 \mathrm{mmol} / \mathrm{l}$ & $3.4-4.6 \mathrm{mmol} / \mathrm{l}$ \\
Sodium & $151 \mathrm{mmol} / \mathrm{l}$ & $147-156 \mathrm{mmol} / \mathrm{l}$ \\
Total proteins & $6.3 \mathrm{~g} / \mathrm{dl}$ & $5.4-8.2 \mathrm{~g} / \mathrm{dl}$ \\
\hline
\end{tabular}

non-septic exudate (mixed cell types, moderate cellularity, predominance of non-degenerate neutrophils without phagocytized bacteria, presence of foamy macrophages, mature lymphocytes and, occasionally, reactive mesothelial cells), probably as a consequence of an increased permeability secondary to inflammation and vascular damage.

The cat was hospitalized and started oxygen therapy. Additionally, intravenous (IV) crystalloid fluid therapy with potassium chloride was given to correct the dehydration and provide fluid therapy maintenance. Antibiotic treatment with ceftriaxone $(25 \mathrm{mg} / \mathrm{kg}$, IV, twice a day [BID]) along with doxycycline $(10 \mathrm{mg} / \mathrm{kg}$, orally [PO], once a day [SID], for 21 days) was started, and midazolam $(0.2 \mathrm{mg} / \mathrm{kg}$, intramuscularly [IM], SID) was given to increase appetite. Due to the low haematocrit and the low haemoglobin concentration, a red cell concentrate was administered to increase the supply of oxygen to the tissues.

Whole blood in EDTA was molecularly screened for agents of infectious feline anaemia. DNA was extracted with High Pure PCR Template Preparation Kit (Roche Diagnostics $\mathrm{GmbH}$, Germany). A PCR with primers Mycop F1 and Mycop Rev1 for detection of Mycoplasmatales, including the genus Mycoplasma (Portuguese Institute for Accreditation [IPAC] accredited test ref. PT03.12; Biopremier, Portugal), yielded a negative result. The amplification program was as follows: an initial step at $94{ }^{\circ} \mathrm{C}$ for $2 \mathrm{~min}, 45$ cycles of $30 \mathrm{~s}$ at $94{ }^{\circ} \mathrm{C}, 30 \mathrm{~s}$ at $57{ }^{\circ} \mathrm{C}$ and $1 \mathrm{~min}$ at $72{ }^{\circ} \mathrm{C}$, and final extension at $72{ }^{\circ} \mathrm{C}$ for $5 \mathrm{~min}$. The PCR kit HaemoTicks (ref. BIOV IA 48; Biopremier, Portugal) was used for the detection of Rickettsiales (including the genera Anaplasma, Ehrlichia and Rickettsia), with primers RickO F2 and RickO Rev1; and of Piroplasmorida (comprising the genera Babesia, Cytauxzoon and Theileria), with primers Piro F2 and Piro Rev2. The amplification program was as follows: an initial step at $94{ }^{\circ} \mathrm{C}$ for $2 \mathrm{~min}, 40$ cycles of $30 \mathrm{~s}$ at $94{ }^{\circ} \mathrm{C}, 30 \mathrm{~s}$ at $58.5^{\circ} \mathrm{C}$ and $15 \mathrm{~s}$ at $72{ }^{\circ} \mathrm{C}$, and final extension at $72{ }^{\circ} \mathrm{C}$ for $5 \mathrm{~min}$. A negative result was obtained for Rickettsiales; and a positive result for Piroplasmorida. An amplicon with 743 bp of the $18 \mathrm{~S}$ rRNA gene was sequenced (GenBank accession no. KU710344). A BLAST analysis of the GenBank database (http://www.ncbi.nlm.nih.gov/BLAST/) revealed $99.9 \%$ identity with the only two DNA sequences of $C$. manul available (accession nos. AY485690 and AY485691; corresponding to 1 nucleotide substitution), from two Pallas's cats caught in Mongolia [5]; $100 \%$ identity with sequences of Cytauxzoon sp. (GenBank accession nos. HM146422 and HM146424) from domestic cats sampled in Italy [11]; 99.9 \% identity with sequences of Cytauxzoon sp. (GenBank accession nos. AY309956 and EU622908) from domestic cats sampled in Spain [9] and France [10], 
respectively; and $100 \%$ identity with several other sequences from wild mammals deposited in GenBank also as Cytauxzoon sp., including: AY496273, from an Iberian lynx (Lynx pardinus) sampled in Spain [12]; KT361074, from a wildcat (Felis silvestris) sampled in Romania [14]; and KT361080, from a Eurasian lynx also sampled in Romania [14]. The maximum homology obtained with the second closest species, C. felis, was $96.7 \%$ (corresponding to 23 nucleotide substitutions).

A phylogenetic analysis including the DNA sequence obtained in the present study was performed to compare it with other sequences of Cytauxzoon spp. previously deposited in GenBank. Sequences were aligned with Clustal X version 1.83 (http://www.clustal.org/). A phylogenetic tree (Fig. 1) was computed with PAUP version 4.0b10 (http://paup.csit.fsu.edu/) using the HKY85 model [16] for calculating distances, and neighbour-joining method with 1,000 replications to estimate the node reliability. Gaps were treated as missing data.

Giemsa-stained blood smears were prepared from peripheral blood to check for intraerythrocytic piroplasms by using an $\times 1,000$ magnification, but erythroparasitaemia was not observed. During the subsequent days, the cat's body temperature remained high and haematocrit dropped to $7.6 \%$. After the molecular confirmation of Cytauxzoon sp., the animal was treated with azithromycin (10 mg/kg, SID, for 10 days), as atovaquone was not available. Despite daily monitoring and intensive supportive care, the cat's condition declined over the following ten days and the animal died. No post-mortem pathological examination was feasible.

\section{Discussion}

To the authors' best knowledge, this is the first report of infection with Cytauxzoon sp. (clustering together with C. manul) in a felid from Portugal, as demonstrated by molecular analysis. Previous studies have reported cases of infection with Cytauxzoon sp. in domestic cats from southern Europe, namely Spain, France and Italy [9-11]. In these cases, the detected agent Cytauxzoon sp., although not named as such, was molecularly identical to $C$. manul. The study by Carli et al. [11] reported clinical disease and persistent infection in Italian cats, but most animals out of 115 cats were subclinically infected.

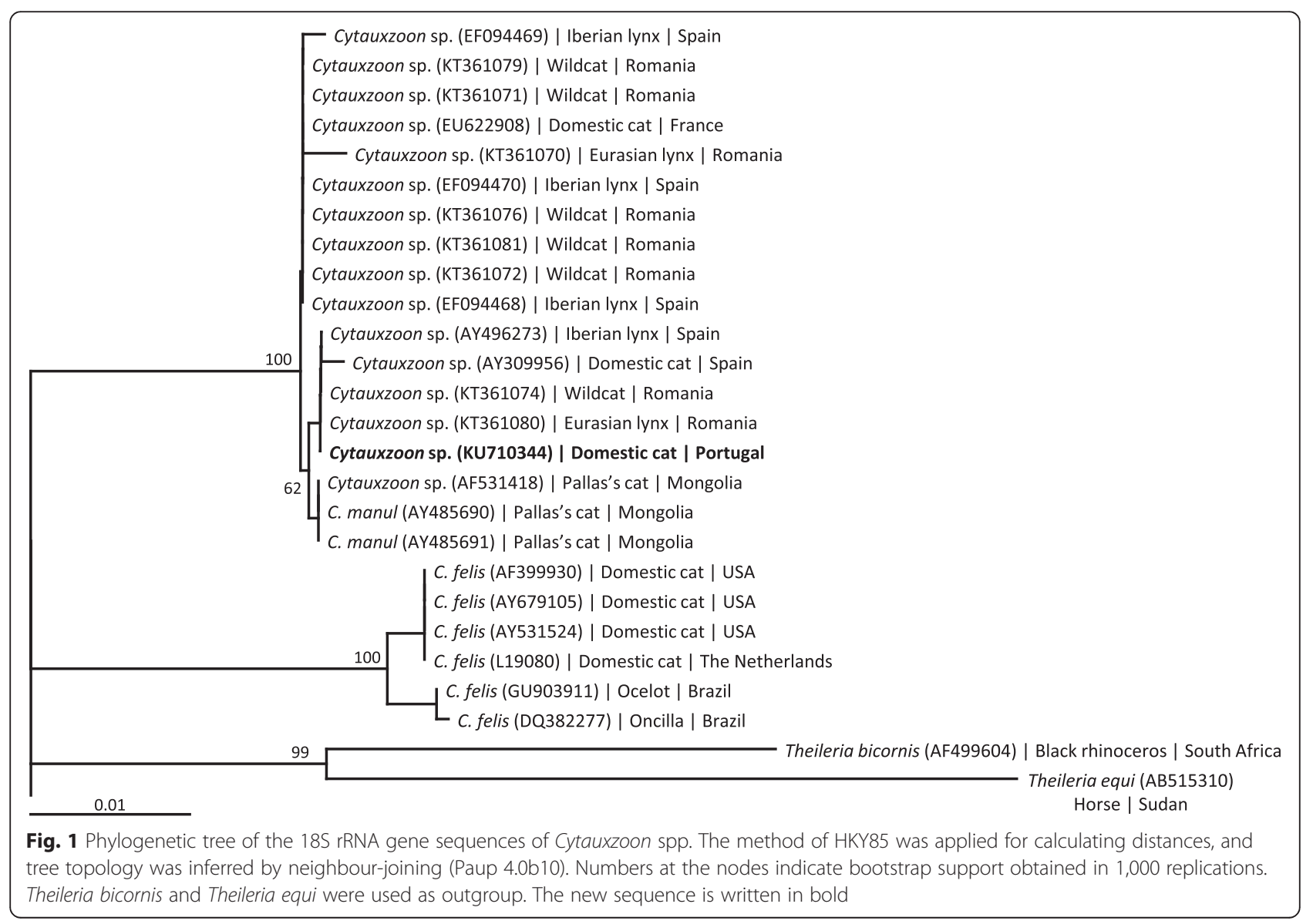


Previously considered a rare disease, cytauxzoonosis due to $C$. felis has now been reported in more than one third of the United States (especially in south-eastern and south-central states) and also in South America and in Europe, with an expected increase in the geographical range due to the ubiquity of tick vectors and their high capacity to adapt to different environments and host species [17-19]. Cytauxzoon manul was reported in Pallas's cats from Mongolia [7] and in captive lions (Panthera leo) in Zimbabwe [8]; and Cytauxzoon sp./C. manul in Eurasian lynxes and wildcats from Romania [14]. Natural infection in domestic cats by C. manul might have involved a species jump from Pallas's cats.

Cytauxzoonosis is usually associated with an outdoor exposure, particularly to unhewn rural areas, where contact with ticks is more frequent $[11,19]$. The most common clinical findings of cytauxzoonosis are anorexia, depression, anaemia, vomiting, icterus and high fever [20]. Pancytopenia, splenomegaly and hepatomegaly are also common. However, there are no pathognomonic findings on $\mathrm{CBC}$, serum chemistry, urinalysis or imaging studies [19]. Such non-specific and wide variety of clinical signs makes it impossible to confirm or exclude this diagnosis based exclusively on a clinical assessment. A conclusive diagnosis can be attained through the visualization of reticuloendothelial cells packed with Cytauxzoon schizonts (basophilic and amorphous protozoal bodies in the cell cytoplasm). Likewise, the visualization of intraerythrocytic piroplasms, i.e. 1-2 $\mu \mathrm{m}$ organisms with light blue cytoplasm and a dark red nucleus (commonly described as "signet ring") in Giemsa-stained peripheral blood smears might help the diagnosis. Confirmation can be done with a more sensitive and specific method, like the PCR, even though it cannot be used to differentiate between acute and chronic cytauxzoonosis. Nevertheless, due to the extremely rapid course of illness associated with this disease, usually with no specific physical findings, a diagnosis is often made only by post-mortem examination. In the present case, the cat was presented with acute lethargy, anorexia and pyrexia, and the rapid course of illness led to death in a few days, with a confirmation of Cytauxzoon sp. by molecular analysis. In previous experimental infection with $C$. manul in domestic cats there was a low parasitaemia, but clinical signs were absent [15]. In the present report, considering the severity of clinical signs, the absence of immunosuppression factors and young age of the cat, we presume that Cytauxzoon sp. clustering together with $C$. manul might be highly virulent in domestic cats.

Cytauxzoonosis by $C$. felis is estimated to cause death in $90 \%$ of the infected cats [20]. For that reason, treatment should be started as soon as possible for all cats clinically suspected of cytauxzoonosis, even if a definitive diagnosis has not been confirmed [19]. The standard of care for cytauxzoonosis appears to be a 10-day course of the antimalarial atovaquone $(15 \mathrm{mg} / \mathrm{kg}, \mathrm{PO}$, three times a day [TID]) combined with the antibiotic azithromycin (10 mg/kg, PO, SID), along with an aggressive supportive therapy for sepsis [21]. However, atovaquone remains an expensive drug, which many owners are not able to afford, and furthermore is hard to get. Even with the recommended therapy, some cats remain persistent carriers of $C$. felis, serving as reservoirs for infection via a tick vector [19]. A recommended therapy could not be found specifically for $C$. manul in the scientific literature.

Amblyomma americanum, the lone star tick, is a highly competent vector for C. felis [22]. Dermacentor variabilis has also been experimentally demonstrated as a vector [23]. As these ticks have so far not been described in Portugal, Rhipicephalus sanguineus (sensu lato), Ixodes spp. or Dermacentor spp. might be hypothesized as being involved in the transmission of Cytauxzoon sp. in the country. Current preventive strategies in regions of endemicity are limited to prophylactic tick control (fipronil in spot-on formulations or imidacloprid/ flumethrin in collars) and keeping cats indoors, in order to reduce exposure to ectoparasites and their transmitted pathogens [24, 25].

\section{Conclusions}

Clinical manifestations along with the molecular analysis support the assumption that domestic cats might be infected with and serve as a reservoir host for Cytauxzoon sp. clustering together with $C$. manul. It is also suggested that interspecies transmission might be more frequent than previously thought. Further studies are needed to improve scientific knowledge on the biology and genetic diversity of this parasite, especially including its vectors, its vertebrate hosts and their geographical range. This report will increase the focus of the veterinary medical community towards $C$. manul in domestic cats.

\section{Ethical approval}

All the clinical procedures in this study were in accordance with the Portuguese legislation for the protection of animals (Decree-Law $n^{\circ} 113 / 2013$ ), as ascertained by the board of Hospital do Gato.

\section{Competing interests}

The authors declare that they have no competing interests.

\section{Authors' contributions}

AMA: examined blood smears, analysed data and drafted the manuscript; JS, CN and MR: performed molecular analysis; MJF and FS: conducted

clinical examination, treatment and follow-up; LMC: reviewed the manuscript; LC: analysed data and revised the manuscript. All authors read and approved the final manuscript. 


\section{Acknowledgements}

AMA holds a PhD fellowship (SFRH/BD/85427/2012) from Foundation for Science and Technology (FCT), Portugal.

Publication of this paper has been sponsored by Bayer Animal Health in the framework of the $11^{\text {th }}$ CVBD World Forum Symposium.

\section{Author details}

'CIISA, Faculty of Veterinary Medicine, Universidade de Lisboa (ULisboa), Lisbon, Portugal. ${ }^{2}$ Biopremier S.A., Campus da Faculdade de Ciências, ULisboa, Lisbon, Portugal. ${ }^{3}$ Hospital do Gato, Lisbon, Portugal. ${ }^{4}$ Department of Veterinary Sciences, School of Agrarian and Veterinary Sciences, University of Trás-os-Montes e Alto Douro (UTAD), Vila Real, Portugal.

Received: 24 February 2016 Accepted: 11 April 2016

Published online: 10 May 2016

\section{References}

1. Beugnet F, Marié JL. Emerging arthropod-borne diseases of companion animals in Europe. Vet Parasitol. 2009;163:298-305.

2. Colwell DD, Dantas-Torres F, Otranto D. Vector-borne parasitic zoonoses: emerging scenarios and new perspectives. Vet Parasitol. 2011;182:14-21.

3. Lloret A, Addie DD, Boucraut-Baralon C, Egberink H, Frymus T, Gruffydd-Jones T, et al. Cytauxzoonosis in cats: ABCD guidelines on prevention and management. J Feline Med Surg. 2015:17:637-41.

4. Brown HM, Berghaus RD, Latimer KS, Britt JO, Rakich PM, Peterson DS. Genetic variability of Cytauxzoon felis from 88 infected domestic cats in Arkansas and Georgia. J Vet Diagn Invest. 2009;21:59-63.

5. Shock BC, Birkenheuer AJ, Patton LL, Olfenbuttel C, Beringer J, Grove DM, et al. Variation in the ITS-1 and ITS-2 rRNA genomic regions of Cytauxzoon felis from bobcats and pumas in the eastern United States and comparison with sequences from domestic cats. Vet Parasitol. 2012;190:29-35.

6. Ketz-Riley CJ, Reichard MV, Van den Bussche RA, Hoover JP, Meinkoth J, Kocan AA. An intraerythrocytic small piroplasm in wild-caught Pallas's cats (Otocolobus manul) from Mongolia. J Wildl Dis. 2003;39:424-30.

7. Reichard MV, Van Den Bussche RA, Meinkoth JH, Hoover JP, Kocan AA A new species of Cytauxzoon from Pallas' cats caught in Mongolia and comments on the systematics and taxonomy of piroplasmids. J Parasitol. 2005;91:420-6

8. Kelly P, Marabini L, Dutlow K, Zhang J, Loftis A, Wang C. Molecular detection of tick-borne pathogens in captive wild felids, Zimbabwe. Parasit Vectors. 2014;7:514.

9. Criado-Fornelio A, Gónzalez-del-Río MA, Buling-Saraña A, Barba-Carretero JC. The "expanding universe" of piroplasms. Vet Parasitol. 2004;119:337-45.

10. Criado-Fornelio A, Buling A, Pingret JL, Etievant M, Boucraut-Baralon C, Alongi $A$, et al. Hemoprotozoa of domestic animals in France: prevalence and molecular characterization. Vet Parasitol. 2009;159:73-6.

11. Carli E, Trotta M, Chinelli R, Drigo M, Sinigoi L, Tosolini P, Furlanello T, Millotti A, Caldin M, Solano-Gallego L. Cytauxzoon sp. infection in the first endemic focus described in domestic cats in Europe. Vet Parasitol. 2012;183(3-4):343-52.

12. Luaces I, Aguirre E, García-Montijano M, Velarde J, Tesouro MA, Sánchez C, et al. First report of an intraerythrocytic small piroplasm in wild Iberian lynx (Lynx pardinus). J Wildl Dis. 2005;41:810-5.

13. Millán J, Naranjo V, Rodríguez A, de la Lastra JM, Mangold AJ, de la Fuente J. Prevalence of infection and $18 \mathrm{~S}$ rRNA gene sequences of Cytauxzoon species in Iberian lynx (Lynx pardinus) in Spain. Parasitology. 2007:134:995-1001.

14. Jirsová D, Mihalca AD, Gherman CL, Dâ Amico G, Qablan MA, Modry D, et al. Cytauxzoon infections in wild felids from Carpathian-Danubian-Pontic space: further evidence for a different Cytauxzoon species in European felids. J Parasitol. In press.

15. Joyner PH, Reichard MV, Meinkoth JH, Milne VE, Confer AW, Kocan AA, et al. Experimental infection of domestic cats (Felis domesticus) with Cytauxzoon manul from Pallas' cats (Otocolobus manul). Vet Parasitol. 2007;146:302-6.

16. Hasegawa M, Kishino $H$, Yano T. Dating of the human-ape splitting by a molecular clock of mitochondrial DNA. J Mol Evol. 1985:22:160-74.

17. Dantas-Torres F, Otranto D. Dogs, cats, parasites, and humans in Brazil: opening the black box. Parasit Vectors. 2014;7:22

18. Rizzi TE, Reichard MV, Cohn LA, Birkenheuer AJ, Taylor JD, Meinkoth JH. Prevalence of Cytauxzoon felis infection in healthy cats from enzootic areas in Arkansas, Missouri, and Oklahoma. Parasit Vectors. 2015;8:13.
19. Sherrill MK, Cohn LA. Cytauxzoonosis: diagnosis and treatment of an emerging disease. J Feline Med Surg. 2015:17:940-8.

20. Birkenheuer AJ, Le JA, Valenzisi AM, Tucker MD, Levy MG, Breitschwerdt EB. Cytauxzoon felis infection in cats in the mid-Atlantic states: 34 cases (1998-2004). J Am Vet Med Assoc. 2006;228:568-71.

21. Cohn LA, Birkenheuer AJ, Brunker JD, Ratcliff ER, Craig AW. Efficacy of atovaquone and azithromycin or imidocarb dipropionate in cats with acute cytauxzoonosis. J Vet Intern Med. 2011;25:55-60.

22. Reichard MV, Edwards AC, Meinkoth JH, Snider TA, Meinkoth KR, Heinz RE, et al. Confirmation of Amblyomma americanum (Acari: Ixodidae) as a vector for Cytauxzoon felis (Piroplasmorida: Theileriidae) to domestic cats. J Med Entomol. 2010;47:890-6

23. Blouin EF, Kocan AA, Glenn BL, Kocan KM, Hair JA. Transmission of Cytauxzoon felis Kier, 1979 from bobcats, Felis rufus (Schreber), to domestic cats by Dermacentor variabilis (Say). J Wildl Dis. 1984;20:241-2

24. Reichard MV, Thomas JE, Arther RG, Hostetler JA, Raetzel KL, Meinkoth JH, et al. Efficacy of an imidacloprid $10 \%$ /flumethrin $4.5 \%$ collar (Seresto, Bayer) for preventing the transmission of Cytauxzoon felis to domestic cats by Amblyomma americanum. Parasitol Res. 2013;112 Suppl 1:11-20.

25. Coles TB, Dryden MW. Insecticide/acaricide resistance in fleas and ticks infesting dogs and cats. Parasit Vectors. 2014;7:8.

\section{Submit your next manuscript to BioMed Central and we will help you at every step:}

- We accept pre-submission inquiries

- Our selector tool helps you to find the most relevant journal

- We provide round the clock customer support

- Convenient online submission

- Thorough peer review

- Inclusion in PubMed and all major indexing services

- Maximum visibility for your research

Submit your manuscript at www.biomedcentral.com/submit
Biomed Central 